\title{
BORN VALUES FOR VECTOR AND TENSOR ASYMMETRIES IN ELECTRON-DEUTERON SCATTERING
}

\author{
V.I. Zhaba* \\ Uzhhorod National University, 88000 Uzhhorod, Ukraine, Voloshin Str. 54
}

(Received March 13, 2019)

\begin{abstract}
Using the previously obtained analytic form factors for the deuteron wave function in the coordinate representation for the nucleon-nucleon potential Argonne v18, are calculated the Born values for vector $A_{B}^{L}, A_{B}^{T}$ and tensor $A_{B}^{L L}$, $A_{B}^{T T}, A_{B}^{L T}$ asymmetries, which are necessary for estimating radiative corrections to polarization observables in elastic electron-deuteron scattering in lepton variables. The momentum-angular dependence for vector and tensor asymmetries is illustrated in 3D format. Each component of the asymmetry has its own peculiarity of the form depending on the values of the scattering angle or momentum of the particle.
\end{abstract}

PACS: 03.65.Nk, 13.40.Gp, 13.88.+e, 21.45.Bc

\section{INTRODUCTION}

A deuteron can be used as a target for an electron beam or as a particle that is scattered on a proton and nuclei. For example, in paper [1], results are presented for spin-dependent electron scattering on polarized protons and deuterons for the BLAST experiment, carried out at the MIT-Bates Linear Accelerator Center. The paper [2] presents the results of a study for spin observables in $d p$ - scattering and testing $T$-invariance in applying the modified Glauber theory. In [3] the full set of deuteron analyzing powers in elastic $d p$-scattering at $190 \mathrm{MeV} /$ nucleon is indicated. In [4] proton and deuteron analyzing powers and 10 spin correlation coefficients were measured for elastic $\mathrm{p}+\mathrm{d}$ scattering at an energy of bombarding protons of 135 and $200 \mathrm{MeV}$. In this paper, analytical forms of DWF are used for theoretical calculations of the Born values of vector and tensor asymmetries, which are necessary for estimating radiative corrections to polarization observables in elastic electrondeuteron scattering in lepton variables [5]. For numerical calculations used the realistic phenomenological potential of the Argonne group - Argonne v18.

\section{THE VECTOR AND TENSOR ASYMMETRIES}

The task of studying the lepton radiative corrections in elastic electron-deuteron scattering remains relevant in recent years [6].

In order to obtain formulas for radiative corrections to the polarization observables for the reaction $e^{-}\left(k_{1}\right)+d\left(p_{1}\right) \rightarrow e^{-}\left(k_{2}\right)+d\left(p_{2}\right)$, it is necessary to parameterize the state of polarization of the target in the definitions of the 4-moment of the particles in this reaction [5]. The 4 -vector $s_{\mu}$ of deuteron polarization and the quadrupole polarization tensor $p_{\mu \nu}$ describe the polarization state of the target. For the polarization state, this parametrization depends on the directions along which the longitudinal and transverse polarization components of the deuteron in the fixed frame are determined. The quantity $s_{\mu}$ describes the vector polarization of the deuteron.

Five Born values of vector $A_{B}^{L}, A_{B}^{T}$ and tensor $A_{B}^{L L}, A_{B}^{T T}, A_{B}^{L T}$ asymmetries were considered when searching for radiative corrections to polarization observables in elastic ed- scattering in leptonic variables [5].

The spin-dependent parts of the cross-section are determined by the vector polarization of the initial deuteron and by the longitudinal polarization of the electron beam $[5,7]$

$$
\begin{array}{r}
\frac{d \sigma_{B}^{L}}{d p^{2}}=-\frac{\pi \alpha^{2}}{4 \tau V^{2}} \frac{2-\rho}{\rho} \sqrt{\rho(4 \tau+\rho)} G_{M}^{2} ; \\
\frac{d \sigma_{B}^{T}}{d p^{2}}=-\frac{\pi \alpha^{2}}{V p^{2}} \sqrt{\frac{(4 \tau+\rho) c}{\tau}} G_{M} G,
\end{array}
$$

where

$$
\begin{array}{r}
G=2 G_{C}+\frac{2}{3} \eta G_{Q} ; \quad c=1-\rho-\rho \tau ; \\
\eta=\frac{P^{2}}{4 M_{D}^{2}} ; \quad \rho=\frac{p^{2}}{V} ; \quad \tau=\frac{M_{D}^{2}}{V} .
\end{array}
$$

In the laboratory system, these expressions for the cross-sections allow one to write values for asymmetries (or the spin correlation coefficients) in elastic ed-scattering in the Born approximation [7]

$$
\begin{aligned}
& \frac{d \sigma_{B}^{L}}{d p^{2}}=\frac{\pi}{\varepsilon_{2}^{2}} \sigma_{N S} \sqrt{(1+\eta)\left(1+\eta \sin ^{2} \varphi\right)} \tan \varphi \csc \varphi G_{M}^{2} \\
& \frac{d \sigma_{B}^{T}}{d p^{2}}=2 \frac{\pi}{\varepsilon_{2}^{2}} \sigma_{N S} \sqrt{\eta(1+\eta)} \tan \varphi G_{M}\left(G_{C}+\frac{\eta}{3} G_{Q}\right)
\end{aligned}
$$

*Corresponding author E-mail address: victorzh@meta.ua 
where $\varepsilon_{2}$ are the energy of the scattered electron; $G_{C}(p), G_{Q}(p), G_{M}(p)$ are deuteron form factors; $\varphi=\theta_{e} / 2 ; \theta_{e}$ is the electron scattering angle.

These two vector asymmetries are formed due to the vector polarization of the deuteron target (respectively, the longitudinal and transverse directions of the spin 4-vectors) and the longitudinal polarization of the electron beam [5]

$$
\begin{aligned}
& A_{B}^{L}=-\eta \sqrt{(1+\eta)\left(1+\eta \sin ^{2} \varphi\right)} \tan \varphi \csc \varphi G_{M}^{2} I_{0}^{-1} \\
& A_{B}^{T}=-2 \sqrt{\eta(1+\eta)} \tan \varphi G_{M}\left(G_{C}+\frac{\eta}{3} G_{Q}\right) I_{0}^{-1}
\end{aligned}
$$

where

$$
I_{0}=A+B \tan ^{2} \varphi .
$$

The ratio between the vector longitudinal and vector transverse polarization asymmetries is written in the form [7]

$$
\frac{A_{B}^{L}}{A_{B}^{T}}=\frac{d \sigma_{B}^{L} / d \sigma_{B}}{d \sigma_{B}^{T} / d \sigma_{B}}=\frac{2-\rho}{4} \sqrt{\frac{\rho}{c \tau}} \frac{G_{M}}{G}
$$

or in the laboratory system [5]

$$
\frac{A_{B}^{L}}{A_{B}^{T}}=\sqrt{\eta\left(1+\eta \sin ^{2} \varphi\right)} \csc \varphi \frac{G_{M}}{G} .
$$

The 4-vector for a tensor-polarized deuteron target is written as

$$
s_{\mu}^{(I)}=\frac{2 \varepsilon_{\mu \lambda \rho \sigma} p_{1 \lambda} k_{1 \rho} k_{2 \sigma}}{V \sqrt{V c \rho}} \text { at } I=L, T, N .
$$

In the Born approximation, part of the crosssection depends on the tensor polarization of the deuteron target $[5,7]$

$\frac{d \sigma_{B}^{p}}{d p^{2}}=\frac{d \sigma_{B}^{L L}}{d p^{2}} R_{L L}+\frac{d \sigma_{B}^{T T}}{d p^{2}}\left(R_{T T}-R_{N N}\right)+\frac{d \sigma_{B}^{L T}}{d p^{2}} R_{L T}$

where are the three components for this section:

$$
\begin{gathered}
\frac{d \sigma_{B}^{L L}}{d p^{2}}=\frac{\pi \alpha^{2}}{p^{4}} 2 c \eta \times \\
\times\left\{8 G_{C} G_{Q}+\frac{8}{3} \eta G_{Q}^{2}+\frac{2 c+4 \tau \rho+\rho^{2}}{2 c} G_{M}^{2}\right\} \\
\frac{d \sigma_{B}^{T T}}{d p^{2}}=\frac{\pi \alpha^{2}}{p^{4}} 2 c \eta G_{M}^{2} \\
\frac{d \sigma_{B}^{L T}}{d p^{2}}=\frac{\pi \alpha^{2}}{p^{4}} 4 \eta(2-\rho) \sqrt{\frac{c \rho}{\tau}} G_{Q} G_{M} .
\end{gathered}
$$

In the laboratory system these expressions for the cross-sections lead to the following three tensor asymmetries (or analyzing capabilities) in elastic edscattering, that were induced by tensor polarization of the deuteron target and the unpolarized electron beam [7]

$\frac{d \sigma_{B}^{p}}{d p^{2}}=\frac{\pi}{\varepsilon_{2}^{2}} \sigma_{N S}\left[S_{L L} R_{L L}+S_{T T}\left(R_{T T}-R_{N N}\right)+S_{L T} R_{L T}\right]$ or this formula is presented in [5] as

$$
I_{0} A_{B}^{p}=A_{B}^{L L} R_{L L}+A_{B}^{T T}\left(R_{T T}-R_{N N}\right)+A_{B}^{L T} R_{L T},
$$

where $A_{B}^{L L}, A_{B}^{T T}, A_{B}^{L T}$ are the tensor polarizations asymmetries:

$$
\begin{gathered}
A_{B}^{L L}=\frac{1}{2}\left\{8 \eta G_{C} G_{Q}+\frac{8}{3} \eta^{2} G_{Q}^{2}+\right. \\
\left.+\eta\left[1+2(1+\eta) \tan ^{2} \varphi\right] G_{M}^{2}\right\} I_{0}^{-1}
\end{gathered}
$$

$$
A_{B}^{T T}=\frac{1}{2} \eta G_{M}^{2} I_{0}^{-1}
$$

$$
A_{B}^{L T}=-4 \eta \sqrt{\eta+\eta^{2} \sin ^{2} \varphi} \sec \varphi G_{Q} G_{M} I_{0}^{-1} .
$$

Between the components $S_{i j}$ and $A_{B}^{i j}$ there is the following relationship $\left(\pi / \varepsilon_{2}^{2}\right) \sigma_{N S} S_{i j}=A_{B}^{i j} / I_{0}$.

\section{CALCULATIONS}

In paper [8] for research of radiative corrections to the polarization observed in elastic ed- scattering in leptonic variables have been calculated the Born values of vector and tensor asymmetries. The deuteron wave functions in coordinate representation for eight nucleon-nucleon potentials (Nijm1, Nijm2, Nijm93, Reid93, Argonne v18, OBEPC, MT and Paris) were applied for numerical calculations of these asymmetries. The momentum-angular dependence of values vector $A_{B}^{i}(p, \theta)$ and tensor $A_{B}^{i j}(p, \theta)$ asymmetries have been also evaluated in $3 \mathrm{D}$ format only for Reid93 potentials. Due to the fact that in [8] the values of the angular dependence of these five asymmetries for the Argonne v18 potential are calculated, therefore, in this paper, only the momentum-angular dependence of the asymmetries will be calculated. The deuteron wave function (DWF) in the coordinate representation for the phenomenological realistic nucleon-nucleon potential Argonne v18 in analytical form [9] is used for numerical calculations

$$
\left\{\begin{array}{c}
u(r)=r^{3 / 2} \sum_{i=1}^{N} A_{i} \exp \left(-a_{i} r^{3}\right) \\
w(r)=r \sum_{i=1}^{N} B_{i} \exp \left(-b_{i} r^{3}\right)
\end{array}\right.
$$

The coefficients of DWF for potential Argonne v18 are given in [9].

In the laboratory system, the Born values of the vector (1), (2) and tensor (5)-(7) asymmetries are determined by the deuteron form factors. In turn, the deuteron form factors depend on the DWF in the coordinate representation (see $[10,11]$ ). 


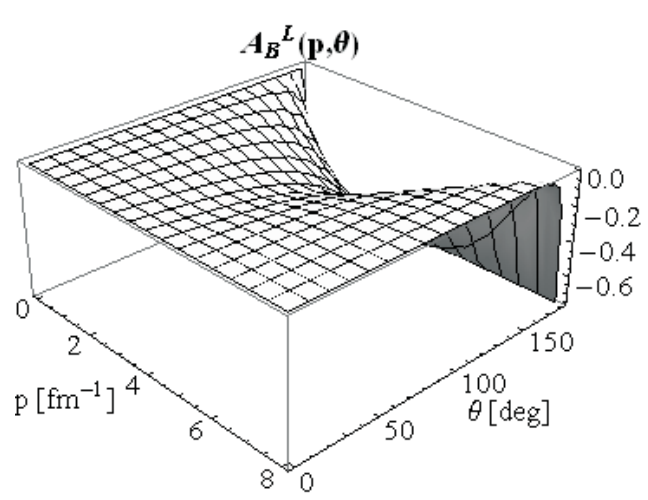

Fig.1. The vector asymmetry

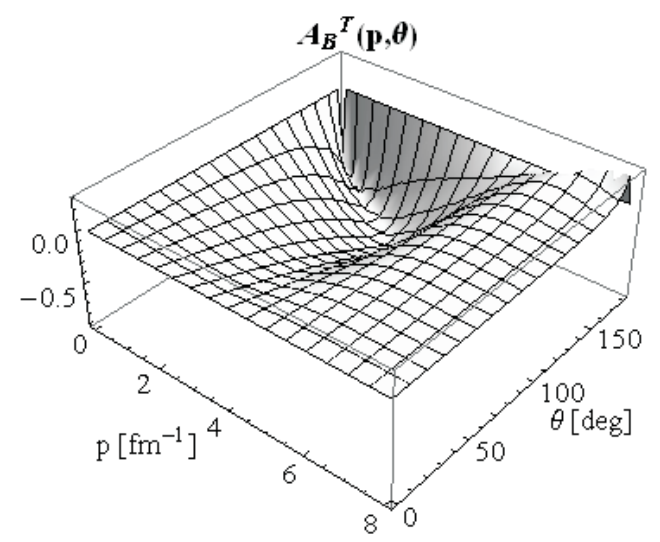

Fig.2. The vector asymmetry

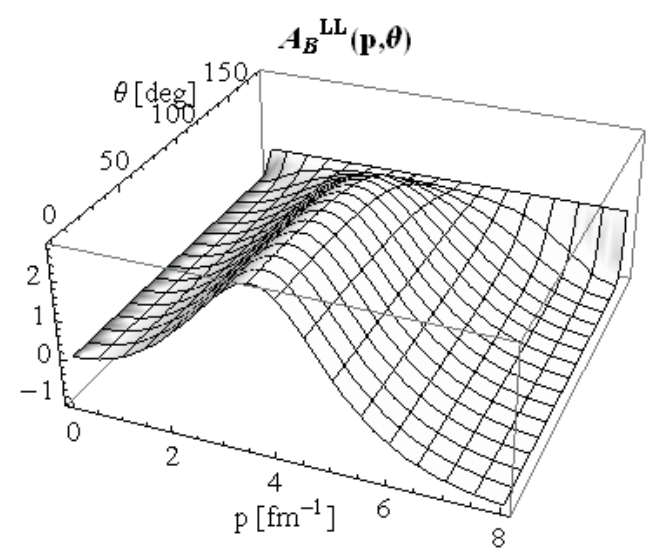

Fig.3. The tensor asymmetries

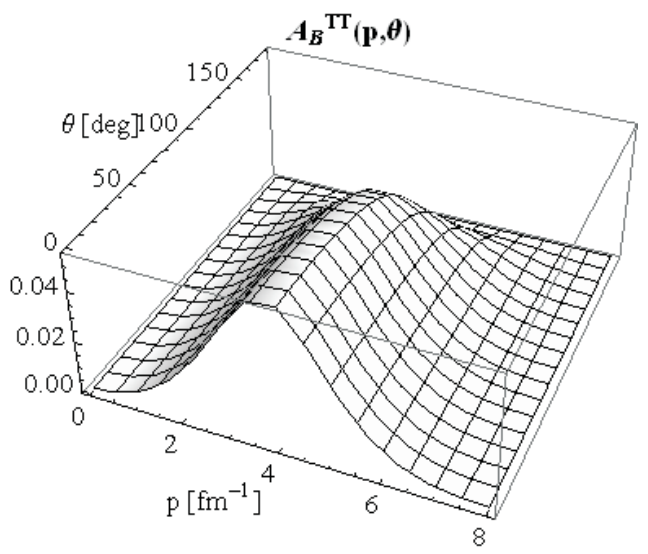

Fig.4. The tensor asymmetries

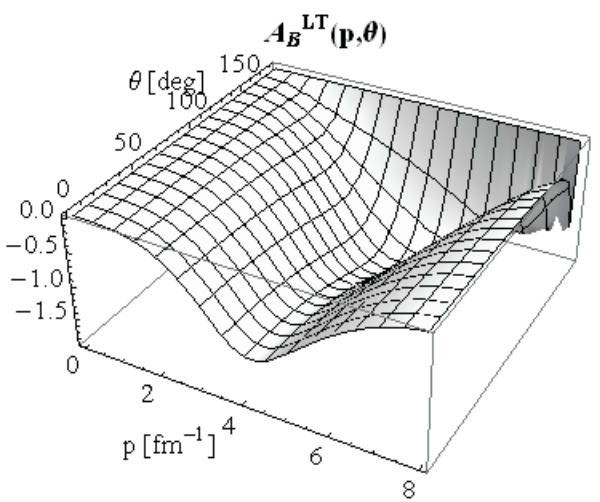

Fig.5. The tensor asymmetries

The Figs.1-5 display momentum-angular dependence in $3 \mathrm{D}$ format for vector $A_{B}^{i}(p, \theta)$ and tensor $A_{B}^{i j}(p, \theta)$ asymmetries, which are calculated for for DWF (8) for Argonne v18 potential.

For vector asymmetry $A_{B}^{L}$ (see Fig.1), the characteristic plane at small angles up to 70 degrees and a rapid decrease at large scattering angles. According to Fig.2 the vector asymmetry $A_{B}^{T}$ forms a kind of "tray".

As seen in Figs.3 and 4 for tensor asymmetries $A_{B}^{L L}$ and $A_{B}^{T T}$ there is a hump (peak) near $3.7 \mathrm{fm}-1$ in the range of angles 0-180 degrees. For the tensor asymmetry $A_{B}^{L T}$ (see Fig.5), on the contrary, there is a pit.

\section{CONCLUSIONS}

The previously obtained coefficients of the analytical form of the deuteron wave function (8) in the coordinate representation for the phenomenological realistic nucleon-nucleon potential Argonne v18 calculated the set of Born values for vector $A_{B}^{L}, A_{B}^{T}$ and tensor $A_{B}^{L L}, A_{B}^{T T}, A_{B}^{L T}$ asymmetries. These values for asymmetries are necessary for the subsequent estimating of radiative corrections to polarization observables in elastic ed- scattering in lepton variables [5].

As shown in Figs.1-5 each component of the vector and tensor asymmetries has its own peculiarity of the form depending on the values of the scattering angle or the momentum of the particle. The aforecited and reviewed vector and tensor asymmetries (the spin correlation coefficients) can be compared with two sets of components for cross-sections [5]:

$$
\begin{array}{r}
\frac{d \sigma_{B}^{\beta}}{d p^{2}}=V_{\beta A}(-\theta) \frac{d \sigma_{B}^{A}}{d p^{2}} \quad \text { at } A=L, \text { T and } \beta=l, t ; \\
\frac{d \sigma_{B}^{\beta}}{d p^{2}}=T_{\beta A}(-\theta) \frac{d \sigma_{B}^{A}}{d p^{2}} \quad \text { at } A=L L, T T \text { and } \beta=l l, t t, l t
\end{array}
$$

for polarization 4-vectors

$$
\begin{gathered}
s_{\mu}^{(l)}=\frac{2 \tau k_{1 \mu}-p_{1 \mu}}{M_{D}} ; \quad s_{\mu}^{(n)}=s_{\mu}^{(N)} \\
s_{\mu}^{(t)}=\frac{k_{2 \mu}-(1-\rho-2 \rho \tau) k_{1 \mu}-\rho p_{1 \mu}}{\sqrt{V c \rho}} .
\end{gathered}
$$

In addition, further studies of polarization observables in elastic lepton-deuteron scattering, including 
lepton masses [12] (and for the case of spin correlation coefficients in the limit of zero lepton mass) are promising.

\section{References}

1. D.K. Hasell et al. Spin-Dependent Electron Scattering from Polarized Protons and Deuterons with the BLAST Experiment at MIT-Bates // Rev. Nucl. Part. Sci. 2011, v.61, p.409.

2. A.A. Temerbayev, Yu.N. Uzikov. Spin Observables in Proton-Deuteron Scattering and TInvariance Test // Phys. Atom. Nucl. 2015, v.78, p.35-42.

3. K. Sekiguchi et al. Complete set of deuteron analyzing powers from dp elastic scattering at $190 \mathrm{MeV} /$ nucleon // Phys. Rev. C. 2017, v.96, p.064001.

4. B.V. Przewoski et al. Analyzing powers and spin correlation coef?cients for $\mathrm{p}+\mathrm{d}$ elastic scattering at 135 and $200 \mathrm{MeV} / /$ Phys. Rev. C. 2006, v.74, p.064003.

5. G.I. Gakh, M.I. Konchatnij, N.P. Merenkov. Radiative Corrections to Polarization Observables in Elastic Electron-Deuteron Scattering in Leptonic Variables // J. Exp. Theor. Phys. 2012, v.115, p.212-236.
6. G.I. Gakh, M.I. Konchatnij, N.P. Merenkov. Leptonic radiative corrections to elastic deuteronelectron scattering // Phys. Rev. C. 2018, v.98, p.045212.

7. G.I. Gakh, N.P. Merenkov. Radiative Corrections to Polarization Observables in Elastic ElectronDeuteron Scattering in Hadronic Variables // $J$. Exp. Theor. Phys. 2004, v.98, p.853-869.

8. V.I.Zhaba. The vector and tensor asymmetries and deuteron wave function for different nucleonnucleon potentials // World Scientific News. 2018, v. 114, p.230-240.

9. V.I. Zhaba. New analytical forms of wave function in coordinate space and tensor polarization of deuteron // Mod. Phys. Lett. A. 2016, v.31, p.1650139.

10. F. Gross. Relativistic Calculation of the Deuteron Electromagnetic Form Factor. II* // Phys. Rev. 1964, v.136, p.B140-B161.

11. R. Gilman, F. Gross. Electromagnetic structure of the deuteron // J. Phys. G. 2002, v.28, p.R37R116.

12. G.I. Gakh, A.G. Gakh, E. Tomasi-Gustafsson. Polarization observables in lepton-deuteron elastic scattering including the lepton mass // Phys. Rev. C. 2014, v.90, p.064901.

\section{БОРНОВСКИЕ ЗНАЧЕНИЯ ВЕКТОРНЫХ И ТЕНЗОРНЫХ АСИММЕТРИЙ В ЭЛЕКТРОН-ДЕЙТРОННОМ РАССЕЯНИИ}

\section{В. И. Жаба}

С использованием ранее полученньх коэффициентов аналитической формы волновой функции дейтрона в координатном представлении для нуклон-нуклонного потенциала Argonne v18 рассчитаны борновские значения векторных $A_{B}^{L}, A_{B}^{T}$ и тензорных $A_{B}^{L L}, A_{B}^{T T}, A_{B}^{L T}$ асимметрий, необходимые для оценки радиационных поправок к поляризационным наблюдаемым в упругом электрон-дейтронном рассеянии в лептонных переменных. Импульсно-угловая зависимость для векторных и тензорных асимметрий проиллюстрирована в формате 3D. Каждая компонента асимметрии имеет свою особенность формы в зависимости от значений угла рассеяния или импульса частицы.

\section{БОРНІВСЬКІ ЗНАЧЕННЯ ВЕКТОРНИХ І ТЕНЗОРНИХ АСИМЕТРІЙ В ЕЛЕКТРОН-ДЕЙТРОННОМУ РОЗСІЯННІ}

\section{B. І. ЖКаба}

3 використанням раніше отриманих коефіцієнтів аналітичної форми хвильової функції дейтрона в координатному представленні для нуклон-нуклонного потенціалу Argonne v18 розраховані борнівські значення векторних $A_{B}^{L}, A_{B}^{T}$ і тензорних $A_{B}^{L L}, A_{B}^{T T}, A_{B}^{L T}$ асиметрій, необхідні для оцінки радіаційних поправок до поляризаційних спостережуваних у пружному електрон-дейтронному розсіянні в лептонних змінних. Імпульсно-кутова залежність для векторних і тензорних асиметрій проілюстрована в форматі 3D. Кожна компонента асиметрії має свою особливість форми в залежності від значень кута розсіяння або імпульсу частинки. 